\title{
Stroke in patients with COVID-19 diagnosis in a Public National Hospital
}

\begin{abstract}
Infection by the new coronavirus (SARS-CoV-2) causes a multisystemic disorder, being the main manifestations in the respiratory system. However, neurological manifestations have been reported showing an increased incidence of ischemic or hemorrhagic stroke.

A retrospective study (January, February and March 2021), in the database of "Hospital Nacional El Salvador" in order to detect and describe the findings in admitted patients who required Brain Computed Tomography (CT) scan due to Acute Neurological manifestations during their hospitalization.

Objective: To describe de incidence of stroke in COVID-19 patient admitted to Hospital El Salvador compare to El Salvador population.

Methods: Observational cross-sectional study in January, February and March 2021 at "Hospital Nacional El Salvador" designated as COVID Hospital to face the Pandemic. The epidemiological data, images, and reports from the Radiology Department of all patients admitted to the hospital were obtained via the electronic database "Patient Care Integral System" ("Sistema Integral de Atención al Paciente" - SIAP). The following definitions were stablished: 1 . Normal CT, 2. Abnormal CT; highlighting the presence of ischemic or hemorrhagic stroke. The Study was approved by the Research Ethics Committee.

Results: During the period studied, a total of 3,632 patients with clinical diagnosis of COVID-19 were registered. CT scan was required for $87(2.4 \%)$ patients, of whom 74 $(2.04 \%)$ presented some type of abnormality; 20 (27.02\%) of the abnormal CT scans had findings corresponding to a stroke (ischemic or hemorrhagic); this represents an incidence of $0.55 \%$ of the total patients assisted in this period. The age-adjusted global mortality rate from cerebrovascular diseases (2014, updated 2018) is 25.2 per 100,000 inhabitants; our finding would indicate an increase in the expected rate of stroke compared to general population.
\end{abstract}

Conclusion: Coinciding with other reports, we found that the disease COVID-19 has a higher incidence of stroke in hospitalized patients older than 60 years.
Volume II Issue 4 - 202I

Cruz de Pereira Susana Yanira,' Bortoli Romina Georgina, ${ }^{2}$ Camputaro Luis Alberto, ${ }^{3}$ Tamayo Ortiz Jose Luis, ${ }^{4}$ Bello Quesada Manuel Enrique, ${ }^{5}$ Ruiz Zelaya Francisco, ${ }^{6}$ Bonilla Carranza Rudy Armando, ${ }^{7}$ Vigne Cuellar Paolo Antoine ${ }^{8}$

'Head of Radiology Service. Hospital Nacional El Salvador ${ }^{2}$ Intensive Care Department. Hospital Nacional El Salvador. San Salvador-El Salvador

${ }^{3}$ Head Teaching and Research Department. Hospital Nacional El Salvador. San Salvador-El Salvador

${ }^{4}$ Intensive Care Department. Hospital Nacional El Salvador. San Salvador-El Salvador

${ }^{5}$ Head Intensive Care Department. Hospital Nacional El Salvador. San Salvador-El Salvador

6Intensive Care Coordinator. Intensive Care Department. Hospital Nacional El Salvador. San Salvador-El Salvador ${ }^{7}$ Vice principal. Hospital Nacional El Salvador. San Salvador-El Salvador

${ }^{8}$ Student, fifth year medical degree. Universidad Jose Matias Delgado. San Salvador-El Salvador

Correspondence: Luis Alberto Camputaro, Head Teaching and Research Department, Hospital Nacional El Salvador. Av de la Revolucion 222. San Salvador, El Salvador, Tel +503 60I I-307I, Email luis.camputaro@gmail.com

Received: June 30, 2021 | Published: July 26, 2021

Keywords: COVID-19, stroke, incidence, computed tomography

\section{Introduction}

The infection caused by coronavirus-2 (SARS-CoV-2) was declared a pandemic on March 11, 2020, ${ }^{1}$ according to WHO data, a total of 166,346,635 cases of COVID-19 have been reported until May 24, 2021, including 3,449,117 deaths. In El Salvador, WHO reports the 72,220 cases and 2,211 deaths. $^{2}$

SARS-CoV-2 is an RNA virus, which through its spike protein binds to the angiotensin-converting enzyme (ACE-2) receptor on endothelial cells and smooth muscle., ${ }^{3,4}$ By binding to the ACE-2 receptor, it crosses the cell membrane for replication. ACE-2 is not only expressed in the respiratory system but also in the kidney, small intestine, liver, central nervous system and the immune system. ${ }^{5}$

The greatest expression of coronavirus infection is produced in the respiratory system, where it induces damage to the alveolar-capillary membrane, generating inflammatory factors with alveolar edema and its consequent loss of surface tension and alveoli collapse, which are responsible for the clinical symptoms of patients in respiratory failure due to COVID- $19 .{ }^{6}$
However, SARS-CoV-2 also affects other organs including the brain, which can lead to an ischemic or hemorrhagic stroke. Infection of the central nervous system can occur either by hematogenous, lymphatic or neuronal dissemination. ${ }^{7}$

The prothrombotic state caused by COVID-19 has been widely demonstrated. Several pathophysiological mechanisms have been proposed to explain the higher incidence of thrombotic events, including stroke. One of the principal mechanisms is endothelial damage by SARS-Cov-2, this can be direct through the ACE-2 receptor on vascular endothelial cells, or indirect through the production of inflammatory cytokines, this state is known as COVID-19-associated coagulopathy. Hypoxia can also intervene in the neurologic damage by disrupting the integrity of the blood-brain barrier and producing ischemic damage, leading to a greater risk of bleeding. Furthermore, hypoxia may cause that a previously non-significant intracranial or extracranial arterial occlusion to become symptomatic.

On the other hand, in hemorrhagic stroke, although less frequent, the same mechanisms that increase the risk of ischemic stroke intervene and play an important role in the pathogenesis of cerebral bleeding. 
Likewise, most of the COVID-19 treatment protocols in hospitalized patients include anticoagulant treatment, further increasing the risk of bleeding. At the moment it has not been possible to establish a causal relationship between these two pathologies and what various lines of research maintain is that COVID-19 can act as a trigger or risk factor in a large proportion of cases.

\section{Methods}

Observational cross-sectional study in January, February and March 2021 at Hospital Nacional El Salvador. Data was collected from the electronic database Sistema Integral de Atención al Paciente -SIAP- (Comprehensive Patient Care System); where epidemiological data, images and reports from the Radiology Department of all patients admitted to the Hospital are recorded. We include all patients hospitalized in Hospital El Salvador from January to March 2021 that require a Brain CT scan due to neurological impairment.

The diagnosis of COVID-19 was made through RT-PCR, immunoglobulins in plasma or clinically (ventilator or respiratory failure, positive chest x-ray, fever, persistent cough, anosmia, ageusia). Upon admission all patients received the current treatment protocol, which includes (depending on patient status): anticoagulation (in moderate and severe cases), corticosteroid therapy, convalescent plasma, tocilizumab, remdesivir and antibiotics (if an infection is suspected by clinical or laboratory findings) that are adjusted depending on the results of the antibiotic sensitivity test.

Brain CT imaging studies were obtained from the Radiology Department database, contrasting the findings with the SIAP. The following definitions were stablished: 1 . Normal CT (no abnormal findings), 2. Abnormal CT (any alteration in the normal intracranial structures). Among the abnormal images we describe the prevalence of ischemic or hemorrhagic stroke, global atrophy and non-specific chronic lesions defined as other findings (among others). Qualitative data is expressed in Mean and SD, the proportions in percentage.

\section{Results}

During the period studied, a total of 3,632 patients diagnosed with COVID-19 were registered. A brain CT study was requested for acute neurological syndrome in 87 patients $(2.4 \%), 51 \%$ men and $49 \%$ women, with an age of $70.4 \pm 14.5$ (r 35-94). Out of these an abnormal CT scan was found in 74 patients $(85 \%)$ of which 20 (27\%) were diagnosed with acute stroke (ischemic or hemorrhagic) (Table 1).

Table I Computed tomography scan findings

\begin{tabular}{lllll}
\hline & & Man & Woman & Total \\
\hline Normal & 7 & 6 & $13(14 \%)$ \\
Abnormal & Global Atrophy & 20 & 23 & $43(49 \%)$ \\
& Ischemia & 8 & 6 & $14(16 \%)$ \\
& Hemorrhage & 3 & 3 & $6(6 \%)$ \\
& Others & 6 & 5 & $11(13 \%)$ \\
& & & & $87(100 \%)$
\end{tabular}

In patients with abnormal CT (including stroke) the ages were different compared to patients with normal CT, a higher presence of stroke was found in those over 60 years old (Table 2).
Table 2 Ages Abnormal CT (including stroke) vs Normal CT

\begin{tabular}{lll}
\hline & $\begin{array}{l}\text { Ages } \\
\text { (Mean, SD) }\end{array}$ & Sex \\
\hline Normal & $41.8 \pm 15.1$ & Mens 36.8 \pm 18.8 \\
& & Women $47.5 \pm 7.03$ \\
Abnormal (CVA) & $73.2 \pm 12.4$ & Mens 73.9 \pm 8.09 \\
Abnormal (no CVA) & $75.5 \pm 10.8$ & Mens 76.3 511.8 \\
& & Women 74.7 79.4
\end{tabular}

The results obtained in admitted patients were compared with the PLISA database (America Public Health Information Platform of the Pan American Health Organization/ World Health Organization) with regards to the age adjusted Global Mortality Rate of Cerebrovascular disease (CVD) (2014, updated 2018). ${ }^{8}$ This rate shows CVD as causal in 25.3 per 100,000 inhabitants.

\section{Discussion}

Our findings correspond with the results of previous studies. The evidence to date shows a low incidence of stroke in patients with COVID-19 (1-1.4\%) but with particular characteristics such as: younger affected population, mostly men, higher incidence of severe stroke and presents more frequently occlusion of a large artery. ${ }^{9}$

Stroke as a complication of COVID-19 tends to appear within the first 10 days after admission, with a higher incidence in seriously ill patients, who required ICU care and mechanical ventilation. A patient with SARS-CoV-2 infection is predisposed to develop various stroke forms: atherosclerosis of large arteries (35.2\%), cryptogenic $(32.4 \%)$, cardioembolic (14.1), small vessel disease $(8,4 \%)$ and other causes $(9.9 \%)$. The stroke origins depend on the predominant pathophysiological cause. In patients with a recognized cause of stroke usual cardiovascular risk factors are identified, not being present in cryptogenic stroke patients. ${ }^{9-11}$

Our study has several limitations, the principals are inherent of a retrospective study without a control group, leading to a risk of bias and underdiagnosis of stroke. The diagnosis can be missed, as neurological deterioration in critically ill patients is often attributed to the decline systemic clinical status, hypoxia or hemodynamic instability. Likewise access to a thorough neurological examination is limited especially in ventilated patients under sedation.

The risk of overdiagnosis of COVID-19 is present because PCR test was not performed systematically to every admitted patient. Moreover, characteristic clinical features, radiological findings, IgM and $\mathrm{IgG}$ were also used to establish diagnosis. It is important to note that "Hospital El Salvador" was created exclusively to care patients undergoing Covid-19 disease, therefore, there were limited diagnostic tools to recognize the stroke source at the date of the study. Data regarding the presence of cardiovascular risk in patients included in the study could not be collected. Although the higher incidence of cerebrovascular events is evident in hospitalized patients compared to the general population, we cannot establish the viral infection as a direct cause of stroke.

The findings in our study correlate with those of previous publications, regarding of the low incidence of this complication 
in hospitalized patients with COVID-19, higher incidence in male patients, and the age range affected (between 60 and 70 years).

\section{Conclusion}

The population over 60 years with abnormal CT scan in the presence of an acute neurological event has a higher incidence of stroke compared to the general population. Considering that in 2018 the CVD rate was 25.2 per 100,000 inhabitants (Men 26.7, Women 24.0 ), the incidence of $0.55 \%$ (550 per 100.00 inhabitant) in our hospital population is much higher than the expected.

\section{Acknowledgments}

None.

\section{Conflicts of interest}

The authors declare no conflicts of interest.

\section{References}

1. Cucinotta D, Vanelli M. WHO declares COVID-19 a pandemic. Acta Bio Medica: Atenei Parmensis. 2020;91(1):157-160.

2. World Health Organization (WHO). Coronavirus disease (COVID-19) Panel de control de coronavirus (COVID-19) de la OMS. WHO; 2021.
3. Li W, Moore M, Vasilieva N, et al. Angiotensin-converting enzyme 2 is a functional receptor for the SARS coronavirus. Nature. 2003;426(6965):450-454.

4. Wu F, Zhao S, Yu B, et al. A new coronavirus associated with human respiratory disease in China. Nature. 2020;579:265-269.

5. Ou X, Liu Y, Lei X, et al. Characterization of spike glycoprotein of SARSCoV-2 on virus entry and its immune cross-reactivity with SARS-CoV. Nat Commun. 2020;11:1620.

6. Mateos EA. Armando el Rompecabezas Fisiopatológico del COVID-19. An Fac Cienc Méd. (Asunción). 2020;53(2):105-126.

7. Li YC, Bai WZ, Hashikawa T. The neuroinvasive potential of SARSCoV2 may play a role in the respiratory failure of COVID-19 patients. Journal of medical virology. 2020;(6):552-555.

8. Visualización de Indicadores. PLISA. Plataforma de información en Salud Publica para las Américas. Organización Panamericana de la Salud. Organización Mundial de la Salud.

9. Nannoni S, de Groot R, Bell S, et al. Stroke in COVID-19: a systematic review and meta-analysis. Int J Stroke. 2021;16(2):137-149.

10. Yaghi S, Ishida K, Torres J, et al. SARS-CoV-2 and Stroke in a New York Healthcare System. Stroke. 2020;51(7):2002-2011.

11. Bhatia R, Pedapati R, Komakula S, et al. Stroke in Coronavirus Disease 2019: A Systematic Review. Journal of Stroke. 2020;22(3):324-335. 